\title{
CRÉDITOS Y TRANSFERENCIAS: UNA REFLEXIÓN EN TORNO A LA EXPANSIÓN DEL CONSUMO EN AMÉRICA LATINA
}

\author{
Credits and transfers: a reflection on the consumption expansion in Latin \\ America
}

\author{
Florencia Chahbenderian'
}

\begin{abstract}
Resumen
Uno de los cambios que emergen a la luz del Siglo XXI es la generalización y masificación de nuevas formas de gestión de la pobreza y de la expansión del consumo en diversos países del Sur Global. Los gobiernos latinoamericanos vienen efectuando intervenciones similares basadas en el otorgamiento masivo de Programas de Transferencias Condicionadas de Ingresos. Por otro lado, se vienen implementando diversas medidas de incentivo al consumo, entre ellas se destaca el otorgamiento de créditos para el consumo.

El presente se propone analizar, de modo preliminar, las relaciones existentes entre las políticas sociales y los créditos para el consumo en América Latina en la actualidad, de modo de describir el crédito y el consumo como condiciones de "contener" el conflicto.

Para ello, en primer lugar se introducen algunas nociones teóricas de las que partimos para abordar el análisis propuesto. En segundo término, proponemos una reflexión en torno a las políticas sociales y, particularmente en América Latina, sobre los Programas de Transferencias Condicionadas de Ingresos y su función en el capitalismo actual. En tercer lugar, se presentan algunos datos sobre la expansión financiera y el lugar de los créditos para el consumo en la dinámica de acumulación de capital a nivel regional. Luego se analiza la actual relación entre los créditos y los programas de transferencias con las políticas sociales en América Latina y, por último, se esbozan una serie de reflexiones finales en torno a la función estructural de ambas medidas como pacificadoras del conflicto.
\end{abstract}

Palabras clave: crédito; políticas sociales; consumo; programas de transferencias condicionadas de ingreso; América Latina.

\begin{abstract}
One of the emerging changes in the light of the XXI Century is the generalization and massification of new forms of poverty management and the expansion of consumption in several countries of the Global South. Latin American Governments have been carrying out similar interventions based on the mass granting of Conditional Cash Transfer Programs. On the other hand, various incentive measures for consumption have been implemented, among which the granting of credits for consumption stands out. The article intends to analyze, in a preliminary way, the existing relations between social policies and credits for consumption in Latin America at present, in order to describe the credit and consumption as conditions of "containing" the conflict.
\end{abstract}

\footnotetext{
1 Doutoranda em Sociologia vinculada ao Instituto de Investigação Gino Germani, da Universidade de Buenos Aires. E-mail: florenciachabe@gmail.com.
} 
To do this, we first introduce some theoretical notions from which we started to address the proposed analysis. Secondly, we propose a reflection on social policies and, particularly in Latin America, on the Conditional Cash Transfers Programs and their function in current capitalism. Third, some data are presented on the financial expansion and the place of credits for consumption in the dynamics of capital accumulation at the regional level. Then, the current relationship between credits and transfer programs with social policies in Latin America is analyzed and, finally, a series of final reflections on the structural function of both measures as conflict reducers are outlined.

Keywords: credit; social politics; consumption; conditional cash transfer programs; Latin America.

\section{Introducción}

Uno de los cambios que emergen a la luz del Siglo XXI es la generalización y masificación de nuevas formas de gestión de la pobreza y de la expansión del consumo en diversos países del Sur Global. Los gobiernos latinoamericanos vienen efectuando intervenciones similares basadas en el otorgamiento masivo de dinero a través de Programas de Transferencias Condicionadas de Ingresos. Por otro lado, sobre todo a partir del último decenio, tanto a nivel estatal como privado, se vienen implementando diversas medidas de incentivo al consumo, entre ellas se destaca el otorgamiento de créditos para el consumo.

El presente se propone analizar, de modo preliminar, las relaciones existentes entre las politicas sociales y los créditos para el consumo en América Latina en la actualidad, de modo de describir el crédito y el consumo adoptados por los sujetos, como condiciones de "contener" el conflicto.

Para ello, el artículo se organiza de la siguiente manera. En primer lugar se introducen algunas nociones teóricas de las que partimos para abordar el análisis propuesto. En segundo término, proponemos una reflexión en torno a las politicas sociales y, particularmente en América Latina, sobre los Programas de Transferencias Condicionadas de Ingresos y su función en el capitalismo actual. En tercer lugar, se presentan algunos datos sobre la expansión financiera y el lugar de los créditos para el consumo en la dinámica de acumulación de capital a nivel regional. Luego se analiza la actual relación entre los créditos y los programas de transferencias con las políticas sociales en América Latina y, por último, se 
esbozan una serie de reflexiones finales en torno a la función estructural de ambas medidas como pacificadoras del conflicto.

\section{A modo de apertura: Una aproximación a la situación del capitalismo actual en América Latina}

En el presente partimos de un diagnóstico en el que la situación actual de dominación capitalista a nivel mundial puede caracterizarse a partir de tres aristas: i) un proceso de expropiación excedentaria de recursos vitales (como el agua, aire, tierra y energía), ii) el fortalecimiento de la militarización y represión por parte de los Estados y a nivel internacional, y iii) el desarrollo y gestión de dispositivos de regulación de las sensaciones y mecanismos de soportabilidad social (SCRIBANO, 2012).

Las mutaciones del modo de producción capitalista, y las relaciones sociales que involucra, están en consonancia con los requerimientos de las fases de expansión del capital en cada momento histórico. Desde la perspectiva aquí propuesta, es posible problematizar las transformaciones en la constitución de las relaciones sociales capitalistas, y la re-producción y dis-posición de cuerpos y emociones, en apariencia considerados como lo más intimo e individual (SCRIBANO; VERGARA MATTAR, 2009). En este sentido, las condiciones materiales de existencia de los sujetos son resultado de las tensiones entre diversas maneras de sentirse en un cuerpo. Dicha percepción involucra las lógicas de dominación necesarias para el mantenimiento del orden social elaborado y aceptado y, en esta dirección, es posible observar las conexiones que se establecen entre depredaciónexpropiación y coagulación-licuación de la acción (SCRIBANO, 2012).

Desde el Centro de Investigaciones y Estudios Sociológicos venimos insistiendo en la necesidad de re-pensar la situación actual del capitalismo en el Sur Global, y particularmente en nuestras sociedades latinoamericanas, a partir de una sociología de los cuerpos/emociones ${ }^{2}$ (SCRIBANO, 2012). En esta dirección, las distancias que cada sociedad establece sobre los cuerpos, cómo se los marca, y en qué medida se hallan

\footnotetext{
2 La barra que se inscribe entre cuerpos/emociones hace referencia a que no es posible indagar y reflexionar sobre los cuerpos y las emociones por separado, como si existiera la posibilidad de que unos no remitieran a las otras, y viceversa (SCRIBANO, 2012).
} 
disponibles sus energias sociales, nos aproxima a los mecanismos de dominación que operan en dicha sociedad. "Así, la política de los cuerpos, es decir, las estrategias que una sociedad acepta para dar respuesta a la disponibilidad social de los individuos es un capítulo, y no el menor, de la estructuración del poder. Dichas estrategias se anudan y "fortalecen" por las políticas de las emociones tendientes a regular la construcción de la sensibilidad social.” (SCRIBANO, 2012, p. 102).

A su vez, señalamos la necesidad de construir mediaciones para aprehender la realidad social desde una mirada post-colonial, ${ }^{3}$ en el afán por contribuir a una ciencia social contra-hegemónica, que se permita observar más allá de los horizontes de comprensión de la lógica académica de su época.

En esta dirección, la economía política de la moral nos permite aproximarnos al estudio de aquellas "prácticas de dominación/explotación devenidas principios morales en un momento de los procesos de estructuración social” (SCRIBANO, 2015a, p. 10). En este sentido, el análisis del disfrute inmediato a través del consumo, los sentidos materiales y simbólicos involucrados, así como las sensibilidades en torno a dichas prácticas, nos aportan algunas pistas para aprehender cómo opera la economía política de la moral en la actualidad. Dicha regulación se vuelve fundamental en tanto posibilita tres aspectos centrales: i) que se produzcan sujetos para objetos, con la expansión de las leyes de las cosas a las normas de las relaciones humanas; ii) que dichas prácticas se transformen en imperativos morales (¡disfrútalo!); y iii) establecer la superficie de inscripción cognitivo/afectiva para "la aceptación de la universalización de un interés particular" (SCRIBANO, 2015a, p. 11).

En lo que sigue presentamos dos grandes incentivos al consumo: los programas de transferencias condicionadas de ingreso y los créditos para el consumo. Ambos vienen siendo implementados masivamente por los gobiernos

\footnotetext{
3 "Existen diversas maneras de sistematizar las orientaciones teóricas en las que se fundan los estudios sobre los cuerpos y emociones. Una posible, teniendo en cuenta el contexto latinoamericano y sin pretensiones de exhaustividad, es la siguiente: a) una linea de trabajo ligada a Foucault y sus conceptos de control, disciplinamiento y tecnologias del yo; b) un enfoque conectado a Bourdieu y sus nociones de habitus, hexis corporal y espacio social; c) un conjunto de investigaciones en el campo de lo biopolítico que refieren a Esposito, Agamben, por un lado, y a Negri y Hardt, por otro; y d) las indagaciones que, desde una visión post-colonial, retoman a la corporalidad como pista para un pensamiento contra-hegemónico." (SCRIBANO y VERGARA MATTAR, 2009, p. 412).
} 
latinoamericanos (de forma directa o indirecta) durante los últimos veinte años, además de ser promovidos por los organismos multilaterales de crédito.

\section{Las políticas sociales: el caso de los PTCI}

Una posible aproximación teórica al estudio de las políticas sociales

Con el desarrollo de los Estados modernos y la instauración del capital como relación social hegemónica, nace la idea de ciudadanía basada en sujetos libres e iguales. Aquí emerge una contradicción entre el derecho a la libertad, la igualdad y la propiedad, y la dependencia y desigualdad entre poseedores y desposeídos de los medios de producción, en tanto fundamentos del modo de producción capitalista. Así, "[1]os procesos de desigualdad y expulsión generados en la estructuración de una sociedad basada en la mercantilización de la vida provoca quiebres conflictuales que deben ser subsanados sistémicamente" (DE SENA; MONA, 2014, p. 10). En este contexto, la "cuestión social" busca suturar estas "fallas" sistémicas, interviniendo en la reproducción social a través de las políticas sociales.

Si bien existe una larga tradición de discusión teórica sobre las políticas sociales, y son amplios los debates en torno a su definición, podemos entenderlas preliminarmente como “... aquellas específicas intervenciones sociales del Estado que se orientan (en el sentido de que producen y moldean) directamente a las condiciones de vida y de reproducción de la vida de distintos sectores y grupos sociales, y que lo hacen operando especialmente en el momento de la distribución secundaria del ingreso" (DANANI, 2004, p. 11).

No sólo resultan relevantes por implicar la demarcación de un ámbito de intervención y la definición de un problema considerado público que reviste cierto interés político, sino porque además involucran pujas teóricas y políticas dentro del ámbito estatal y por fuera (por ejemplo, en ámbitos académicos) en torno a los modos de abordaje de dicha problemática (DE SENA; CENA, 2014; GRASSI, 2003). Estas intervenciones parten de concepciones ideológicas que se plasman en los diseños normativos e institucionales de una politica pública determinada (RAMACCIOTTI, 2010), que siempre - de forma más o menos explícita - presentan cierta carga moral. 
En este marco, las politicas sociales adquieren una relevancia fundamental para la producción y reproducción social ya que, para soportar la desigualdad, es necesario generar un conjunto de politicas de los cuerpos/emociones (SCRIBANO, 2012; DE SENA; CENA, 2014). Tal como hemos señalado en otro lado (CENA, CHAHBENDERIAN, DETTANO, 2016), algunas de las principales vinculaciones entre las politicas sociales y las politicas de los cuerpos/emociones se pueden pensar en torno a, al menos, cuatro ejes: i) afectan los niveles de conflictividad social (HALPERIN WEISBURD et al., 2011; OFFE, 1993; SCRIBANO; DE SENA, 2013); ii) construyen sociabilidades (DANANI, 2004), vivencialidades y sensibilidades (DE SENA; CENA, 2014); iii) son performativas de lo social (GRASSI, 2003); y conforman un aspecto central del modo de regulación social y político (NEFFA, 2006; CENA, 2015).

A continuación presentamos brevemente algunas de las principales características del caso bajo estudio: los Programas de Transferencias Condicionadas de Ingreso (PTCI) implementados en Latinoamérica.

Los PTCl como respuestas individualizadas

Durante los últimos veinte años, los PTCI se han posicionado como los programas sociales de "combate" a la pobreza más utilizados por los gobiernos latinoamericanos, expandiéndose no sólo en cantidad (por su masividad), sino también por su extensión a un creciente número de países de la región y del Sur Global. Estos programas fueron (y son) promovidos y en muchos casos financiados por organismos multilaterales de crédito. Si bien según el país y el momento histórico cada programa presenta características peculiares a nivel local, 4 en general es posible identificar cierta continuidad entre un programa y otro, y entre un país y otro, dado que cuentan con un esquema similar, basado fundamentalmente en tres grandes etapas operativas:

\footnotetext{
${ }_{4}^{4}$ Dado el objetivo del presente y por cuestiones de extensión no podemos detenernos en describir cada uno de los programas. Para identificar los PTCI que se vienen implementando en América Latina se puede consultar la Base de Datos de Protección Social no Contributiva de CEPAL (ver: http://dds.cepal.org/bdptc/\#es. Fecha de consulta, 23/10/2017).
} 
- Focalización: tienen requisitos de acceso y permanencia basados en características de los hogares o personas;

- Transferencias: otorgan un ingreso mensual, a través de diversas modalidades (efectivo, depósito en una cuenta bancaria, cheques, etc.);

- Condicionalidades: consideradas corresponsabilidades, reúnen ciertos requisitos que los beneficiarios deben demostrar para recibir la transferencia y permanecer en el programa. Generalmente se vinculan con exigencias en materia de asistencia escolar, nutrición y controles de salud.

En los últimos quince años, al menos 34 países han instrumentado cerca de 57 programas de este tipo, que han atendido cerca de 180 millones de destinatarios. Si observamos su distribución por región, América Latina y el Caribe cuenta con 115,3 millones de destinatarios, Asia y el Pacífico con 61,3 millones, Europa 3,7 y África 0,3 (BBVA, 2011).

Según datos del BID, en 2010 alcanzaron a 129 millones de personas en 18 países de América Latina y el Caribe, representando el 24\% de la población de la región (PAES-SOUSA et al., 2013). Estos datos, si bien no son muy recientes, nos permiten tener una dimensión de la importancia que vienen cobrando estos programas en la región, dada su masividad (DE SENA, 2014).

Son varios los autores que, desde diversas posturas teóricas, vienen señalando que los PTCI se erigen como respuestas estatales individualizadoras (MERKLEN, 2013; LUCIANI, 2010; CENA, 2014; CENA; CHAHBENDERIAN, 2015). Puesto que, al centrar el otorgamiento y la permanencia en características específicas de los sujetos miembros de un hogar, y al exigir ciertos comportamientos por su parte - en pos de lograr la "activación" de la voluntad individual - la "cuestión social" es entendida desde estos programas como una "cuestión individual", constituyéndose como un "apoyo público a la responsabilidad privada” (LAVINAS, 2014, p. 6).

Siguiendo a Cena (2014), al analizar la estructura de estos programas, las soluciones propuestas se basan en el aumento del capital humano de los sujetos pobres, lo cual "ha contribuido a la definición de la situación problema a abordar, a fin de promover que los sujetos sean agentes de sus 
condiciones de carencias, e individualizar, en tanto diagnóstico, las causas de las condiciones de carencias mediante la autorresponsabilización de los destinatarios" (CENA, 2014, p. 7). Por ende, esta lógica subyacente al diseño de los PTCI ubica la responsabilidad de las situaciones de pobreza y marginalidad en los propios sujetos que las vivencian, desechando cualquier explicación "estructural" del problema que intenta abordar individualizando y despolitizando la "cuestión social" (CENA; CHAHBENDERIAN, 2015; FALEIROS, 2004). Según Castro Orellana, "[a]sistiriamos a una suerte de

privatización de las contradicciones, donde los sujetos tienden a experimentar los conflictos estructurales de la sociedad como asuntos que corresponde resolver en el ámbito de lo personal. De esta forma, se produce una invisibilización de la dimensión social de los problemas y todo "queda atado y bien atado" en la simple docilidad del individuo" (CASTRO ORELLANA, 2007, p. 10).

Ahora bien, ese apoyo público a través de transferencias de dinero habilita al sujeto destinatario el acceso al mercado para que gestione sus riesgos de forma privada. De hecho, en las evaluaciones de impacto se resalta el efecto que tienen los PTCI en términos de aumento del consumo de sus beneficiarios (FISZBEIN; SCHADY, 2009; STAMPINI; TORNAROLLI, 2012; LEVY; SCHADY, 2013 en BID, 2017; CECCHINI; MADARIAGA, 2011) y, por ende, a través del efecto multiplicador operan como catalizadores de la actividad económica. En este punto, cabe preguntarnos por las condiciones en que se da dicho acceso al mercado, en contextos de fuerte vulnerabilidad, informalidad y precariedad (como los latinoamericanos).

A continuación introducimos otro paquete de medidas sistemáticamente orientadas a garantizar el consumo: la ampliación de la oferta de créditos para los sectores "sin garantías".

\section{La masificación del crédito}

La expansión del capital financiero

La evolución del sistema financiero en la región viene experimentando importantes cambios en el nuevo Siglo. Uno de los aspectos fundamentales que señala la literatura sobre el tema es la creciente presencia de un modelo 
basado en la banca minorista, así como la mayor importancia de la banca extranjera.

Desde finales del Siglo XX los instrumentos financieros tomaron un rol preponderante en la economía mundial, motorizado en parte por la globalización y mundialización de la economía. En los setenta se inició una era de expansión financiera, y en los años noventa entraron en vigor reformas orientadas a la liberalización financiera, que llevaron a un involucramiento más moderado del Estado y a una mayor participación del sector privado en la región. En este proceso hubo un aumento de la extranjerización y la concentración bancaria. Esta última aumentó sustancialmente en la última década, "con un incremento en el promedio de activos de los tres bancos más grandes desde $51 \%$ en 2000 a $71 \%$ en 2009" (OCDE, 2013, p. 75).

La industria financiera y la actividad bancaria se han ampliado exponencialmente en el último decenio, sobre todo a partir de los cambios operados luego de la crisis financiera de las subprime en 2008. Esto se reflejó, por ejemplo, en el incremento de las operaciones de préstamos para el consumo. ${ }^{5}$

Desde las ciencias sociales, varios académicos vienen señalando las implicancias de este proceso en términos de prácticas de consumo, construcción de subjetividades y hábitos cotidianos.

Según la perspectiva de Graeber (2009) estamos viviendo bajo un “imperio de la deuda" y, como parte de este proceso de financiarización del capital, muchos países tienen economías impulsadas por deudas de consumo. Siguiendo a Bauman (2008) ninguna sociedad ha estado tan fuertemente endeudada como la contemporánea, tanto a nivel individual como nacional. Si "vivir a crédito" tiene sus placeres, el autor se pregunta: ¿por qué retrasar la gratificación, si podemos tenerla aquí y ahora? Y se responde a sí mismo: "Podemos, por así decirlo, consumir el futuro por adelantado, siempre que quede algo por consumir" (BAUMAN, 2008, p. 44).

\footnotetext{
5 Nos referimos a los créditos personales y familiares para la adquisición de bienes de consumo y financiación de tarjetas de crédito. No hacemos alusión a todos los otros tipos de préstamos: hipotecarios, productivos, etc.
} 
Por su parte, Lazzarato (2012) establece el concepto de "hombre endeudado", manifestando la centralidad de financiarización en las prácticas cotidianas de las personas. Esto produce condiciones materiales de existencia basadas en el endeudamiento, que a su vez promueven determinadas subjetividades asociadas a estas estrategias de consumo. En esta línea, el endeudamiento intensifica los mecanismos de explotación y dominio de manera transversal, en tanto produce una pérdida de futuro para los sujetos endeudados, es decir, de la capacidad de ser portadores de decisión y de efectivas posibilidades de acción. Esta situación habilita un control y manejo de parte de los capitales financieros del tiempo de las personas: al comandar su tiempo, se eliminan las posibilidades de una potencial ruptura.

Estos cambios ocurridos en el sistema bancario latinoamericano afectaron los mecanismos de financiamiento. A raíz de la masificación de los programas de transferencias, se crearon nuevos potenciales clientes, hasta entonces no alcanzados por el mercado financiero. De este modo, se conformaron nichos de mercado que fueron aprovechados por la banca, tanto a nivel internacional como local. Según un informe de OCDE (2013), la expansión del crédito en los países latinoamericanos durante los últimos años se centró principalmente en el otorgamiento de préstamos para el consumo. A continuación presentamos algunos de los argumentos y propuestas que propugnan y acompañan dicha expansión.

La inclusión financiera y los créditos al consumo

Desde diversos sectores académicos, empresas y organismos multilaterales de crédito, se promueve la bancarización en la implementación de políticas públicas. Tal como señala Joseph W. Saunders, CEO y Presidente de Visa: "La moneda digital ofrece beneficios esenciales a los gobiernos, permitiéndoles aumentar su eficiencia, promover la inclusión financiera e impulsar el crecimiento económico. (...) Actualmente, Visa cuenta con más de 5000 programas gubernamentales en 47 países" (REYES, 2016). 
Desde hace ya algunos años, varios PTCI en América Latina comenzaron a operar cambios en sus sistemas de pago. Muchos de ellos pasaron de entregar transferencias en efectivo a depositarlas directamente en cuentas bancarias personales a nombre de los beneficiarios, además de ofrecer distintos servicios financieros. Estas iniciativas son promovidas por los organismos multilaterales de crédito, en tanto fomentan la incorporación de los beneficiarios al sistema financiero formal (CHIAPA; PRINA, 2014).

Este proceso de bancarización de los beneficiarios de PTCI se implementa bajo diversas modalidades en cada país. Según un informe del BID (2016), al mes de julio de 2015, tres países de América Latina habían institucionalizado en sus mecanismos de pago de PTCI el acceso a créditos. Esta es una tendencia creciente, vinculada con la financiarización de la economía señalada anteriormente.

En sintonía con la inclusión y la educación financiera surgieron nuevas modalidades de medición a nivel mundial. Un ejemplo es el Índice Multidimensional de Inclusión Financiera (MIFI), que calcula el grado de inclusión de un sistema financiero en diversas economías y a lo largo del tiempo. Este índice evalúa el uso, el acceso y la calidad a través de 18 indicadores en 140 países (en 2011). Sin embargo, un aspecto que se suele omitir de este tipo de argumentos y mediciones es que la creciente bancarización de la población latinoamericana conlleva mayores niveles de endeudamiento (GDS, 2015).

No sólo los bancos ofrecen créditos a los beneficiarios de PTCI entendidos como un grupo poblacional de bajos ingresos, tradicionalmente no alcanzado por las instituciones financieras - sino que también las cadenas minoristas a nivel nacional o internacional de electrodomésticos, ropa, etc., utilizan opciones de financiamiento a través de tarjetas, hasta prestamistas de carácter local (NAVAJAS, 2012). Estos últimos pueden ser locales que ofrecen créditos personales - muchas veces son sucursales adaptadas para este público de grandes bancos internacionales -, así como financieras que ofrecen sus servicios a través de internet y/o en la vía pública. También es muy usual el endeudamiento a nivel informal y en redes locales, con amigos, familiares, etc. 
Como señala Euromonitor International, el proceso de selección para obtener una tarjeta de un comercio es a menudo menos estricto que el requerido por las marcas internacionales como Visa, Master Card o American Express. ${ }^{6}$ De este modo, emergieron diversos abusos y tácticas depredadoras, puesto que los intereses anuales superaron el $200 \%$ en algunos países, y el refinanciamiento de la deuda era unilateral (GRANADOS, 2015).

A modo de ejemplo, en el caso argentino la Administración Nacional de Seguridad Social (ANSES) a mediados de 2017 comenzó a otorgar créditos a beneficiarios de un PTCI - la Asignación Universal por Hijo (AUH). Se dieron más de un millón de créditos en menos de dos meses. Según representantes de ANSES, la elevada demanda de los titulares de dichas prestaciones se debe a que no pueden acceder a créditos en las entidades bancarias tradicionales y terminaban pagando tasas usurarias a diversos prestamistas (TÉLAM, 2017).

Según una investigación de BSLatam, en la última década el crédito al consumo ha crecido exponencialmente en América Latina, alcanzando un crecimiento del $20 \%$ anual. El informe señala que la tarjeta de crédito fue la gran protagonista. Actualmente, un $22 \%$ de la población adulta en la región dispone de al menos uno de los más de 390 millones de plásticos que inundaron el mercado (GRANADOS, 2015). Según la Unidad Financiera del Fondo Multilateral de Inversiones (Fomin), dependiente del Banco Interamericano de Desarrollo, dicho crecimiento fue exponencial en Brasil, México, Argentina, Chile, Venezuela, Colombia y Perú (GRANADOS, 2015).

Si consideramos la deuda per cápita en tarjetas de crédito entre los países latinoamericanos, Venezuela encabeza el ranking con US\$2.169, al dólar oficial. En segundo lugar, Chile supera a Brasil, donde la deuda por persona llega a US\$ 1.276; seguido por Argentina (US\$ 899 al tipo de cambio oficial), México (US\$ 465), Colombia (US\$ 434) y Perú (US\$ 15,4) (GDS, 2015). Según Euromonitor, la relación entre la deuda y el ingreso de

\footnotetext{
6 Chile y Argentina son los países con mayor número de tarjetas, habiendo cerca de 1.700 plásticos que son exclusivos de tiendas comerciales que otorgan con menos requisitos que los exigidos por los bancos (GDS, 2015).
} 
los hogares de la región es del 31,5\%. El mayor ratio lo tiene Chile $(71,7 \%)$, seguido de Venezuela (59,2\%), Brasil (32\%), México (21,8\%), Colombia $(21,4 \%)$ y Argentina (17,2\%) (GRANADOS, 2015).

Paralelo a los elevados niveles de endeudamiento de los hogares de bajos ingresos en América Latina, emerge la pregunta sobre cómo se compone el consumo en dichos sectores. Según la Base de datos de consumo del Banco Mundial, que centraliza datos sobre los patrones de consumo de los hogares en países en desarrollo, en el caso de los segmentos más bajos de la población de 10 países de la región, la mayor proporción de gasto en consumo se destina a alimentos y gastos del hogar (como por ejemplo electricidad). ${ }^{7}$

Esto abre no pocos interrogantes acerca de las condiciones en las que esos ingresos se vuelcan al crédito y al consumo: ¿Cómo afecta el panorama descripto a sus condiciones materiales de existencia? ¿Cuáles son los impactos de dichas políticas en los presupuestos familiares y en las prácticas de consumo? Estas y otras preguntas exceden los fines del presente trabajo, pero quedan delineadas como futuras líneas de indagación.

\section{Créditos al consumo y transferencias condicionadas de ingreso: vivir "contenidos" en el consumo compensatorio}

Dado lo señalado en el apartado anterior, es notoria la extensión y masividad del consumo como una clara herramienta politica que constituye un rasgo central en el actual proceso de elaboración de sensibilidades, resultando efectiva para contener el conflicto (DE SENA; SCRIBANO, 2014).

La idea de consumo compensatorio es útil para lo que aquí queremos ilustrar. Entendemos por dicho consumo a las consecuencias - directas o indirectas, explícitas o implícitas, deseadas o no - de la acción estatal centrada en el incentivo al consumo como base para la expansión del mercado interno. Es así como el consumo compensatorio se consolida como una forma de construir sensibilidades desde el Estado (DE SENA; SCRIBANO, 2014).

\footnotetext{
7 Datos estimados para 2010. Elaboración propia en base a Banco Mundial. Base de Datos del consumo mundial. Disponible en: https://datos.bancomundial.org/. Fecha de consulta: 11/11/2017.
} 
En esta línea, queremos dejar señaladas algunas de las principales aristas en la reproducción de la cadena beneficiario=consumidor=sujeto de crédito=deudor, en donde el ciudadano se materializa bajo algunas de estas figuras. Esta identidad procesual y dinámica nos permite conectar la idea de subsidio/asistencia con ser sujeto de crédito/deudor como base de la ciudadanía para aproximarnos a la comprensión del proceso basado en vivir asistido para estar contenido.

La financiarización de la vida cotidiana, de la que hemos esbozado algunos rasgos, es un componente fundamental en el proceso aquí descripto. La vida a crédito, donde el endeudamiento es la norma, "[e]s la masificación del hombre blanco clase media norteamericano que tiene su vida en cuotas y que "puede perderlo todo"' (SCRIBANO, 2015b, p. 37).

En esta línea, y considerando el caso bajo estudio, cobrar una transferencia monetaria por un programa social y acceder a ciertos servicios financieros mediante la toma de créditos, constituyen lógicas circulares y expansivas, que conforman eslabones fundamentales de la reproducción del capital. Como ilustra la cita a continuación:

“... la banca y el crédito se convierten asimismo en el medio más poderoso para impulsar la producción capitalista más allá de sus propios límites, y en uno de los vehículos más eficaces de las crisis y de las estafas" (MARX, 2017 [1975], p. 698).

Esta última aseveración de Marx debe ser tenida en cuenta, dado que existen evidencias sobre los abusos y estafas en relación con los términos del endeudamiento. Tal como evidencia Macías González, para el caso de los destinatarios de los PTCI:

"Esta población vulnerable ha sido el nuevo nicho de mercados para bancos, cooperativas y financieras, tiendas departamentales, por diferentes intereses politico-ideológicos y económicos. Sus estrategias financieras están condicionadas por factores estructurales que interactúan con pautas culturales que permean en sus lógicas particulares de comportamiento, donde los ingresos son una condicionante clave" (MACÍAS GONZÁLEZ, 2016, p. 193). 
Como hemos señalado en otro lado (CENA; CHAHBENDERIAN, 2012), garantizar el crédito y el consumo son estrategias públicas de "contención" de las poblaciones en condiciones de pobreza. Estas constituyen las principales politicas sociales de cara al Siglo XXI en América Latina, conforme a su indudable masividad y su rol en tanto gestoras de emociones reguladoras del conflicto. En esta línea, cabe preguntarse: ¿qué hay en las sensibilidades que hace tan soportable la condición de vivir endeudado? Dado lo dicho hasta aquí, podemos pensar que estas políticas, en tanto gestoras de sensibilidades normalizadas, 8 elaboran superficies de inscripción para aceptar "verdades ajustadas a intereses" (SCRIBANO, 2015b, p. 14).

\section{Conclusiones}

Los dos grandes paquetes de medidas presentados aquí denotan cierta circularidad, en forma de un espiral creciente, así como una fuerte complementariedad. Ya que, por un lado, se pone un determinado ingreso en manos de los sujetos considerados "pobres" por los programas (beneficiarios). Dicha relación se encuentra cada vez más mediada por bancos e instituciones financieras (bancarización), que además ponen a disposición de sus usuarios diversos productos financieros. Por otro lado, tanto desde el mercado de créditos al consumo formal como informal, se amplia la oferta de créditos al consumo dirigidos a este segmento de la población (endeudamiento). Es posible identificar aquí la secuencia que se produce con los mecanismos de reproducción social que suturan las desigualdades constitutivas del capitalismo y contienen el conflicto social (asistencia) a través de transferencias condicionadas, que están fuertemente mediadas por la bancarización, lo cual se conecta directamente con el acceso al crédito y el endeudamiento.

De este modo, la ampliación de las acciones estatales se efectiviza vía mercado: el Estado ya no es el único responsable de las políticas públicas (SCRIBANO, 2015b). En este contexto, donde es evidente que los límites entre lo público y lo privado se desdibujan, las sensibilidades - en tanto

\footnotetext{
8 "La normalización puede ser entendida como la estabilización, repetición compulsiva, adecuación nomológica y desconexión contextual del conjunto de relaciones sociales que las prácticas de los individuos adquieren en un tiempo/espacio particular.” (DE SENA y SCRIBANO, 2014, p. 69).
} 
prácticas permeadas por los procesos de estructuración social - son gestionadas tanto por el Estado como por el mercado. De este modo, las políticas públicas crean sensibilidades en conexión directa con las "necesidades" de expansión del capitalismo (DE SENA; SCRIBANO, 2014). Bajo los argumentos de una democratización del consumo, los beneficiarios de PTCI pasan a ser, además, clientes financieros. Como resultado de ambas aristas se conforman beneficiarios $=$ consumidores $=$ sujetos de crédito=deudores, en contextos en los que los sujetos se endeudan para "vivir al dia".

Como tal, en los PTCI, el hecho de ver las causas de la pobreza como "fracasos individuales" implica intervenir sobre personas que necesitan supervisión para lograr cambios conductuales.

A su vez, desde otorgar transferencias hasta garantizar créditos al consumo, dichas intervenciones se basan en la auto-gestión de los riesgos, que implica una privatización de los mismos y una auto-responsabilización de los sujetos. En sintonía con ello, en el presente sostenemos que las principales politicas sociales de principios del Siglo XXI en Latinoamérica se basan en garantizar y expandir el consumo de las poblaciones pobres.

$\mathrm{El}$ mito de la equivalencia ingreso $=$ igualdad $=$ bienestar, en el contexto de Estados limitados como los latinoamericanos, en los que amplios sectores de la población permanecen excluidos de los derechos sociales fundamentales, la expansión de las transferencias y los créditos al consumo dirigidos a estos sectores pueden crear círculos perversos y virtuosamente expansivos (en tanto suturan a la perfección un espiral de expansión capitalista).

En este sentido, y retomando lo dicho anteriormente, la "cuestión social" opera sobre las contradicciones intrinsecas del capitalismo, interviniendo en la reproducción social a través de políticas sociales. Estas impactan directamente en la re-producción del orden imperante y de la vida de algunos sectores poblacionales; actúan sobre y son resultado de los modos de estructuración social, al operar sobre los aspectos simbólicos y cognitivo-afectivos. Como hemos visto a lo largo del artículo, la actual gestión de la cuestión social se basa en el consumo. 
Dados los contextos de depredación de bienes comunes, niveles de pobreza e indigencia elevados, y los procesos de segregación y racialización, la expansión del consumo en condiciones de Sur Global merece una reflexión en torno a los modos en que las sociedades son normalizadas en torno al consumo (SCRIBANO, 2015a). En este sentido, sostenemos la necesidad de seguir indagando en la regulación de los cuerpos/emociones involucrados en políticas orientadas a la estabilización y regulación del conflicto.

\section{Bibliografía}

BAUMAN, Zygmunt. Miedo Liquido: la sociedad contemporánea y sus miedos líquidos. Buenos Aires: Paidós, 2008.

BBVA. Avanza el pago electrónico de programas sociales en América Latina y el Caribe. Observatorio Bancario. Análisis Económico. México: BBVA Research, 2011.

BID. Asi funcionan las transferencias condicionadas. Buenas prácticas a 20 años de su implementación. Banco Interamericano de Desarrollo, 2017a. Disponivel em: https://publications.iadb.org/bitstream/handle/11319/8159/Asifuncionan-las-transferencias-condicionadas.PDF?sequence $=7$. Acesso em: $14 / 11 / 2017$.

\section{Programas de transferencias monetarias condicionadas e} inclusión financiera. Nota técnica IDB-TB-1140. División Protección Social y Salud. Banco Interamericano de Desarrollo, 2016.

Sistemas de pago e inclusión financiera en América Latina: Cómo promover avances desde normativas propicias y buenas prácticas, 2017b. Banco Interamericano de Desarrollo. Fondo Multilateral de Inversiones del Grupo BID. Disponivel em: http://idbdocs.iadb.org/wsdocs/getDocument.aspx?DOCNUM=40854930. Acesso em: 14/11/2017.

CASTRO ORELLANA, Rodrigo. Gubernamentalidad y ciudadania en la sociedad neoliberal. Ponencia presentada en las IV Jornadas de Filosofia Politica, Universidad de Barcelona, 2007.

CECCHINI Simone; MADARIAGA Aldo. Programas de transferencias condicionadas. Balance de la experiencia reciente en América Latina y el Caribe. Santiago de Chile: Naciones Unidas, 2011.

CENA, Rebeca. Politicas sociales, cuerpos y emociones a principios del siglo XIX en Argentina. Convergencia Revista de Ciencias Sociales. n. 69, 2015. 
Programas de transferencias condicionadas de ingresos y programas de empleo en Argentina: entre la responsabilización de los destinatarios y la individuación de la cuestión social. Boletín Cientifico Sapiens Research $\mathrm{N}^{\circ}$ 4, Año 1, 2014.

CENA, Rebeca; CHAHBENDERIAN, Florencia. El abordaje estatal de la pobreza en Programas de Transferencias Monetarias Condicionadas. Revista Latinoamericana de Ciencias Sociales, Niñez y Juventud "Infancias, Instituciones Sociales y Contextos Politicos en América Latina y el Caribe" v. 13, n. 1, 2015.

El crédito y el consumo como "condiciones" de contentar y contener a las poblaciones expulsadas. Boletin Onteaiken, v. 14, 2012.

CENA, Rebeca; CHAHBENDERIAN, Florencia; DETTANO, Andrea. Vinculaciones posibles entre el par inclusión/exclusión social y los Programas de Transferencias Monetarias Condicionadas en Argentina. RBSE - Revista Brasileira de Sociologia da Emoção, v. 15, n. 44, 2016.

CHIAPA, Carlos y PRINA, Silvia. Ahorro, contabilidad mental, ahorro por default y transferencias monetarias condicionadas. Proyecto Capital. En breve $\mathrm{N}^{\circ} 53,2014$.

DANANI, Claudia. El alfiler en la silla: sentidos, proyectos y alternativas en el debate de las políticas sociales y de la economia social. In: DANANI, Claudia. Politica social y economia social. Buenos Aires: Universidad Nacional de General Sarmiento-Fundación OSDE, Altamira, 2004.

DE SENA, Angélica. Promoción de microemprendimentos y políticas sociales: ¿Universalidad, focalización o masividad?, Una discusión no acabada. Pensamento Plural, n. 8, 2014.

DE SENA, Angélica; CENA, Rebeca. ¿Qué son las politicas sociales? Esbozos de respuestas. In: DE SENA, Angélica. Las politicas hechas cuerpo y lo social devenido emoción: lecturas sociológicas de las politicas sociales. Buenos Aires: Estudios Sociológicos Editora, 2014.

DE SENA, Angélica; MONA, Anaclara. A modo de introducción: la cuestión social, las politicas sociales y las emociones. In: DE SENA, Angélica. Las politicas hechas cuerpo y lo social devenido emoción: lecturas sociológicas de las politicas sociales. Buenos Aires: Estudios Sociológicos Editora, 2014.

DE SENA, Angélica; SCRIBANO, Adrián. Consumo Compensatorio: ¿Una nueva forma de construir sensibilidades desde el Estado?. Revista Latinoamericana de Estudios sobre Cuerpos, Emociones y Sociedad (RELACES) $N^{\circ} 15$, Año 6, 2014.

DETTANO, Andrea; CENA, Rebeca; CHAHBENDERIAN, Florencia. ¿Qué significa "estar incluidos"? Un análisis desde los Programas de Transferencias Condicionadas de Ingresos implementados en Argentina en la 
primera década del Siglo XXI. In: SCRIBANO, Adrian; ARANGUREN, Martín. Aportes a una sociologia de los cuerpos y las emociones desde el Sur. Buenos Aires: Estudios Sociológicos Editora, 2017.

FALEIROS, Vicente de Paula. Las funciones de la politica social en el capitalismo. In: BORGIANNI, E. y MONTAÑO, C. La politica Social Hoy. Brasil: Cortez Editora, 2004.

GDS. Crece el endeudamiento de tarjetas de crédito en Latinoamérica [Online]. 2015. Disponivel em: http://www.gdslink.com/crece-elendeudamiento-de-tarjetas-de-credito-en-latinoamerica/. Acesso em: $14 / 11 / 2017$.

GRAEBER, David. Debt: The first five thousand years. The Anarchist Library. 2009.

GRANADOS, Oscar (02/08/2015). América Latina se rinde al consumo [Online], El Pais, 2015. Disponivel em: https://elpais.com/economia/2015/07/30/actualidad/1438260987_77075 3.html. Acesso em: 01/11/2017.

GRASSI, Estela. Politicas y problemas sociales en la sociedad neoliberal: La otra década infame (I). Buenos Aires: Espacio Editorial, 2003.

HALPERIN WEISBURD, L. et al. Problemas de género en la Argentina del siglo XXI: feminización de la pobreza e inequidad del mercado laboral. Cuadernos del CEPED, Núm. 11. Buenos Aires: Centro de Estudios sobre Población, Empleo y Desarrollo, Facultad de Ciencias Económicas, UBA, 2011.

LAVINAS, Lena. La asistencia social en el siglo XXI. New Left Review $\mathrm{N}^{\circ} 84$, 2014.

LAZZARATO, Maurizio. La dette ou le vol du temps. Le monde diplomatique, février 2012. Disponivel em: https://www.mondediplomatique.fr/2012/02/LAZZARATO/47416. Acesso em: 14/11/2017.

LUCIANI, Leandro. La protección social de la niñez: subjetividad y posderechos en la segunda modernidad. Revista Latinoamericana de Ciencias Sociales, Niñez y Juventud, v. 8, n. 2, 2010.

MACÍAS GONZÁLEZ, Gizelle Guadalupe (2016) La monetarización de la pobreza, las mujeres y la revolución microfinanciera en México [Online]. Revista de Estudios de Género. La ventana, n. V (Julio-Diciembre). Disponivel em: http://www.redalyc.org/articulo.oa?id=88446739009. Acesso em: $12 / 11 / 2017$.

MARX, Karl. El Capital: Crítica de la Economía Política. Libro III. México: Siglo Veintiuno, 2017 [1975]. 
MERKLEN, Denis. Las dinámicas contemporáneas de individuación. In: CASTEL R. et. al. Individuación, precariedad, inseguridad. ¿Desinstitucionalización del presente?. Buenos Aires: Paidós, 2013.

NAVAJAS, Sergio. Préstamos de consumo y sobreendeudamiento en América Latina [Online]. 2012. Disponivel em: https://www.fomin.org/enus/Home/News/article-

details/ArtMID/18973/ArticleID/2017/NoTitleLink.aspx. Acesso em: $14 / 11 / 2017$.

NEFFA, José. Evolución conceptual de la Teoría de la Regulación. In: DE LA GARZA TOLEDO, Enrique. Teorias sociales y estudios del trabajo: Nuevos enfoques. México: Anthropos, 2006.

OCDE. La educación financiera en América Latina y el Caribe. Situación actual y perspectivas. Serie políticas públicas y transformación productiva $\mathrm{N}^{\circ}$ 12/2013. Banco de Desarrollo de América Latina, 2013.

OFFE, Clauss. Contradicciones en el Estado de Bienestar. Madrid: Alianza, 1993.

PAES-SOUSA, Romulo, REGALIA, Ferdinando y STAMPINI, Marco. Condiciones para el éxito de la puesta en práctica de programas de transferencias monetarias condicionadas: Lecciones de América Latina y el Caribe para Asia. Banco Interamericano de Desarrollo, División de Protección Social y Salud. Resumen de Políticas, IDB-PB-192, 2013.

RAMACCIOTTI, Karina. Reflexiones en torno a cómo pensar las intervenciones sociales del Estado. Revista de Estudios Maritimos y Sociales, Año 3, N³, 2010.

REYES, Felipe. INCLUSION FINANCIERA: Peru 8vo en Latinoamerica [Online]. 2016. Disponivel em: http://telecomunicacionesperu.blogspot.com.ar/2016/09/inclusion-financiera-peru-8vo-en.html.

Acesso em: 14/11/2017.

SCRIBANO, Adrian. ¡Disfrútalo! Una aproximación a la economía política de la moral desde el consumo. Buenos Aires: El Aleph, 2015a.

SCRIBANO, Adrian. Comienzo del Siglo XXI y Ciencias Sociales: Un rompecabezas posible. Polis $\mathrm{N}^{\circ} 41,2015 \mathrm{~b}$.

SCRIBANO, Adrian. Sociología de los cuerpos/emociones. Revista Latinoamericana de Estudios sobre Cuerpos, Emociones y Sociedad - RELACES. Córdoba, N¹0, Año 4, diciembre 2012-marzo de 2013, 2012.

SCRIBANO, Adrian; DE SENA, Angélica. Los planes de asistencia social en Buenos Aires: una mirada desde los cuerpos y las emociones. Aposta, Revista de Ciencias Sociales, $n^{\circ} 59$, Octubre, Noviembre y Diciembre, 2013. 
SCRIBANO, Adrian; VERGARA MATTAR, Gabriela. FEOS, SUCIOS Y MALOS: la regulación de los cuerpos y las emociones en Norbert Elías. Caderno CRH, v. 22, núm. 56, mayo-agosto, 2009.

TÉLAM. Anses otorgó más de un millón de préstamos personales en casi dos meses [Online], Télam, 2017 Disponivel em: http://www.telam.com.ar/notas/201709/204176-la-anses-otorgo-mas-deun-millon-de-prestamos-personales-en-casi-dos-meses.html. Acesso em: 02/11/2017. 